\title{
Development of C/SiN Hybrid Sealing-Film for Environmental-Cell Window
}

\author{
T. Kawasaki*****, T. Miura*, Z. Cui*, H. Tsutsui**, T. Matsutani** and T. Tanji********
}

* Dept. Electrical Eng., Nagoya Univ., Furo-cho, Chikusa-ku, Nagoya, 464-8603, Japan

** Graduate School of Engineering, Kinki Univ., 3-4-1Higashiosaka, Osaka, 577-8502, Japan

*** EcoTopia Science Inst., Nagoya Univ., Furo-cho, Chikusa-ku, Nagoya, 464-8603, Japan

**** Global Research Center for Environment and Energy based on Nanomaterials Science

(GREEN), 1-2-1 Sengen, Tsukuba, 305-0047, Japan

In-situ controlled-environment TEM (ETEM) is a powerful technique to study nature of materials under the conditions in which they are formed or applied. In cases of actual materials such as catalysts, fuel cells, biological molecules, etc., it is essential to obtain their practical information, not that in laboratory. The authors has been also developed a closed-type environmental-cell (E-cell) system and applied to observations of Au nanoparticulate catalysts [1]. In the above system, window material is one of the most important components because it must seal gases inside the E-cell and its thickness must be thin enough to suppress electron diffraction in the film. Amorphous carbon is suitable to this purpose, because light and conductive element has less influence on illuminated electron beam and thin film $(<10 \mathrm{~nm}$ thick) enabling to withstand atmospheric pressure difference can be obtained [2]. However, carbon films have disadvantage to be easily damaged by the electron beam irradiation under oxygen-containing gas condition. Since silicon nitride $(\mathrm{SiN})$ is relatively stable for the electron beam, it has been applied as another window material [3]. Therefore, hybridization of the carbon and the SiN was expected to show high performance such as less deterioration of imaging resolution, high toughness for pressure difference, and high stability for electron irradiation. In the present paper, we report about $\mathrm{SiN}$ coating on the carbon films with pulsed laser deposition (PLD) technique and results to check its effect.

Figure 1 shows a schematic illustration of the PLD technique for the SiN coating. The target illuminated by the YAG laser was poly-crystalline $\mathrm{Si}_{3} \mathrm{~N}_{4}$. The substrate was carbon thin films supported on dedicated $\mathrm{Cu}$ grids, which were used as E-cell sealing-films in our previous works [1-2]. The SiN was deposited on this carbon film controlling its thickness to an accuracy of $\sim 0.1$ $\mathrm{nm}$ with a quartz oscillator. Structure of the deposited $\mathrm{SiN}$ film was confirmed to be an amorphous with TEM imaging and electron diffraction pattern, as shown in Fig. 2. Composition of the deposited $\mathrm{SiN}$ film was evaluated approximately as $\mathrm{Si}_{3} \mathrm{~N}_{2} \mathrm{O}_{2}$ by X-ray photoelectron spectroscopy (XPS). This means that half the amount of nitrogen was replaced by oxygen in the deposition process, though the vacuum condition of the PLD chamber was $<5 \times 10^{-5} \mathrm{~Pa}$. In order to examine the effect of the coated $\mathrm{SiN}, 200 \mathrm{kV}$ electron beam was irradiated to the sealing-films under the gas environment $(1000 \mathrm{~Pa}$; $\mathrm{CO}+$ Air). As shown in Fig. 3, just the carbon film was 
easily broken and a hole was formed at an area electron beam irradiated until 30 seconds. On the other hand, the hybrid sealing-film had no damage by the irradiation of more than 10 minutes, though contaminated. These results prove that the hybrid sealing-films are more useful for windowed ETEM comparing to the normal carbon films.

\section{References}

[1] T. Kawasaki et al., Rev. Sci. Inst. 80 (2009) 113701

[2] K. Ueda et al., Surf. Interface Anal. 40, 1725 (2008).

¡3† J. F. Creemer et al., Ultramicroscopy 108, 993 (2008).

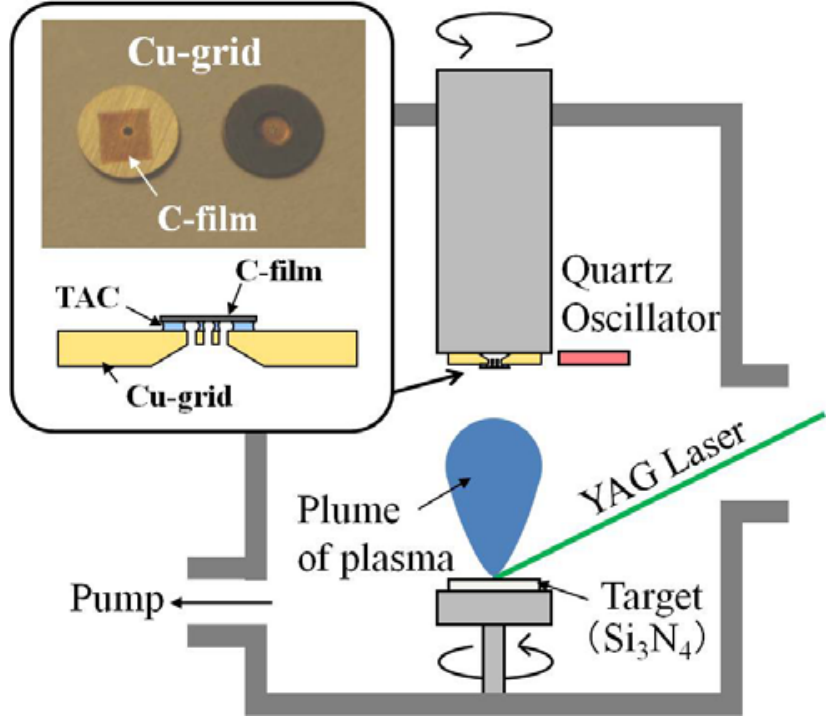

FIG. 1 Schematic illustration of pulsed laser deposition

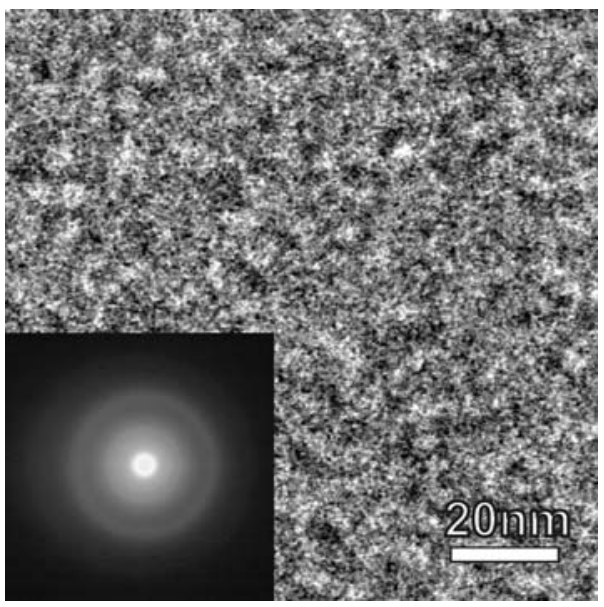

FIG. 2 TEM image and electron diffraction pattern of SiN film deposited with PLD. method for coating of $\mathrm{SiN}$ on $\mathrm{C}$ film supported on $\mathrm{Cu}$ grid.
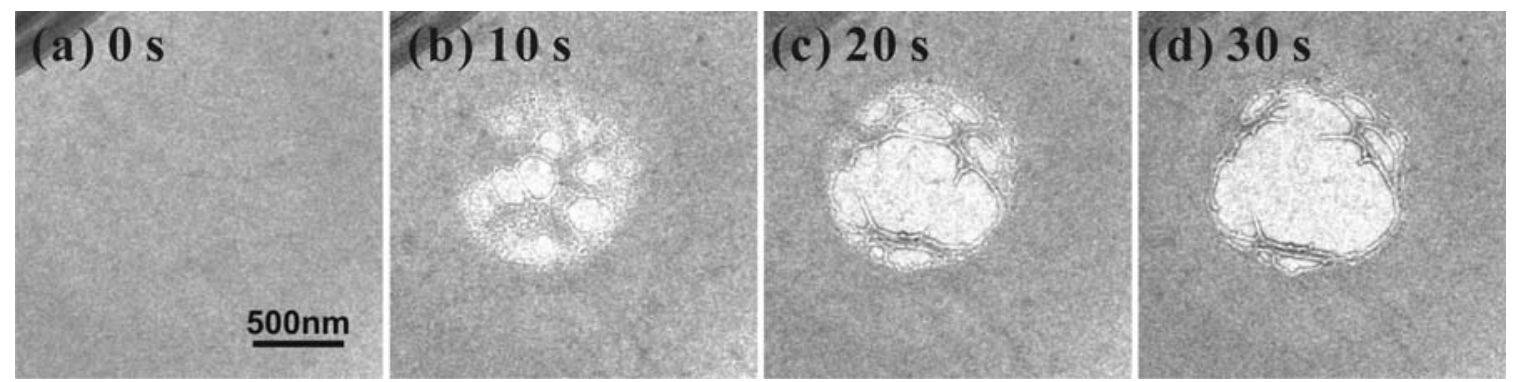

FIG. 3 Effect on a carbon (C; 9nm thick) sealing-film due to electron beam irradiation under reaction gas environment (1000Pa; $\mathrm{CO}+\mathrm{Air})$.
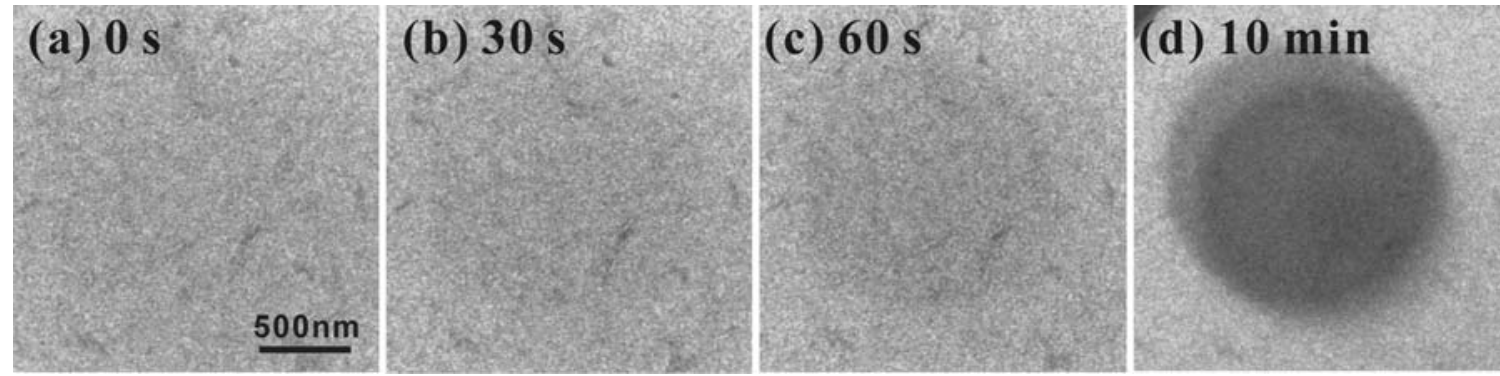

FIG. 4 Effect on a hybrid (C; 9nm + SiN; 2nm) sealing-film under the same condition with FIG. 3. 